\title{
MODELLING IMPLICATIONS \& IMPACTS OF GOING GREEN WITH EV IN SINGAPORE WITH MULTI-AGENT SYSTEMS
}

\author{
Thomas Chee Tat Ho*, Rongshan $\mathrm{Yu}^{*}$, James Russell $\mathrm{Lim}^{\dagger}$ and Pasi Fränti ${ }^{\ddagger}$ \\ *Signal Processing Department, Institute for Infocomm Research, Singapore. \\ E-mail: $\{$ ctho,ryu\}@i2r.a-star.edu.sg \\ ${ }^{\dagger}$ Engineering Science Programme, National University of Singapore, Singapore. \\ E-mail: russell.james@startup.org.sg \\ ${ }^{\ddagger}$ School of Computing, University of Eastern Finland, Finland. \\ E-mail: franti@cs.joensuu.fi
}

\begin{abstract}
As a result of globalization, fossil fuels are being depleted at an alarming rate. Singapore, ranked 9th in the world as net importer of fuels [1], will have to find a more efficient way to utilize them. Over the years, Singapore has been switching its energy dependency on the cheaper natural gas [2]. However, its transportation still depends heavily of petroleum. One of the options to reduce the dependency is to switch to the electric vehicles (EV). However, the impacts and implications of introducing EV into Singapore to its energy distribution system is highly desired before actual deployment, which is very challenging primarily due to lacks of high quality data from reallife scenarios. In this paper, we propose to overcome this issue by using multi-agent system (MAS) based simulation technology to quantify the impacts of EV charging to the electricity system of Singapore.
\end{abstract}

\section{INTRODUCTION}

There is great urgency to improve the efficiency of using this planet's fossil fuels [3]. Due to growing dependency and globalization, it is being alarmingly depleted at a rate faster than imaginable. There has been progress in renewable energy resources. However, it may not be suitable and dependable to support this planet's [4] inhabitants if no effort is made to change the way it is being used.

In Singapore, prevalence of renewable energy resources is low. This is due to intermittent agreeable weather conditions that undermine their reliability. Hence, Singapore relies heavily on fossil fuel imports for its energy needs. In 2011, Singapore ranked 9th in the world as a net importer of crude oil [1]. Therefore, it is even more crucial to adopt better ways to utilize the imports else it will be held ransom by the exacerbating oil prices one day.

Over the years, Singapore has been switching from petroleum to cheaper natural gas power generation [2] for ensuring energy affordability to the masses. However, the one things that still relies heavily on gasoline in Singapore is its transportation; internal combustion engine vehicles (ICEV). Currently in Singapore, there is no cheaper alternative to power ICEV. A natural next step would certainly be switching from ICEV to EV in order to fully enjoy the benefits of using cheaper energy sources including natural gas and other renewable energies.

To electrify the entire transportation is a tremendous effort. There have been numerous feasibility studies on the impacts of EV [5], [6]. Most of such studies used either aggregated or derivatives data of national surveys; leaving much room for improvements into the granularity of information obtained. The rich variations in the data, formerly lost through aggregation, is available only when more levels of details are present. And such richness in the data is what makes the modelling closer to the actual situation. However, it is true that short of having the actual real-life scenario, such data with a full spectrum of the details is a near to impossible task.

Recently, there have been renewed interests in using mutliagent systems (MAS) to obtain more granular information pertaining to certain environments [7], [8], [9]. This paper makes use of MAS to reconstruct the level of details from those national surveys for a more constructive insight to the impacts and implications of introducing EV to Singapore.

\section{Methodology}

\section{A. Multi-Agent System(MATSim)}

In order to understand the traffic plans of Singapore's driving population for this study, it is essential to know the point-to-point locations and the details of the journeys of all drivers' daily routines. Traditionally [10], this can be evaluated using the shortest path algorithm [11] followed by some additive noise to the travel time. This approach only produces an average or an aggregated effect. In addition, it does not fully take into considerations of the traffic congestion, time utilized and drivers' decisions due to driver-to-driver interactions, which are essential to make up the necessary variations for this study.

Recently, there have been many study on using MAS [8], [9] for complex system modelling where the resultant behaviour may be unexpected and hard to foresee. An MAS is essentially a computerized system where multiple agents, perceiving their local information and capable of autonomous decisions, 


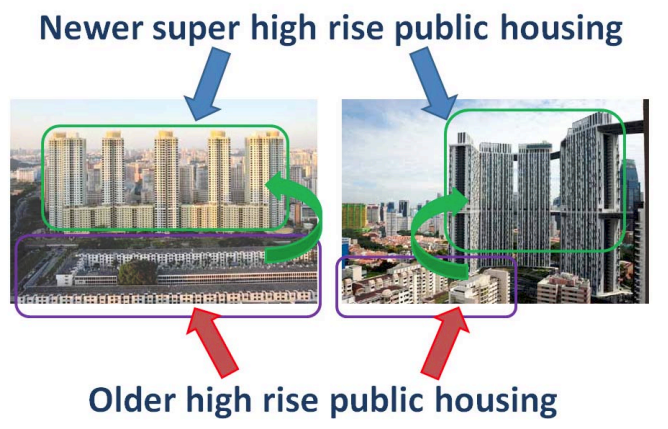

Fig. 1. Transformation of older to newer super high rise public housing to cater to increasing population

interact with each other in a virtual environment [12]. And there have been a number of MAS used to study traffic and its emergent behaviour [13], [14], [15]. In this paper, we use MATSim [16] to simulate the traffic of Singapore. This is mainly because MATSim allows the users to work more on their agents without worrying too much implementation details such as handling interactions among agents, or road network creation.

In order for a simulation to be conducted on MATSim, it requires two inputs:

- Map - the environment

- Plans - the agents' location-to-location plans

The former is essentially a road network input where the drivers, agents in this case, are able to locate their whereabouts and next destinations. The latter refers to the plans of the drivers; the places they intend to travel to during the course of the simulation. Noticed that precise planning of the route to take and when to take it is not required. All of that is left to MATSim's decision.

All input into MATSim are in Extensible Markup Language (XML) format [17]. The map and plans are converted into a data format suitable for MATSim to read as inputs. MATSim outputs two separate sets of data after the simulation; one of which will be used for the energy analysis in this paper.

\section{B. Traffic Plans Based on Singapore's Population}

Singapore, in the 21 st century, is a population of 5.4 million living a small island of $716.1 \mathrm{~km}^{2}$ [18]. Over $80 \%$ of its population live in government subsidized public housing [19]. These are high-rise buildings compromising of residential units placed laterally, above and below one-another. Typically, a single block is able to house from 100 to 200 families. However, given the increase in population in the recent years together with the ever-present land scarcity constraint [20], the housing landscape is slowly being replaced by super high rises capable of housing twice the current amount (Fig. 1).

Singapore is now the third most densely populated country per square kilometres right after Macau and Monaco [21]. The land for residential is already pre-allocated and concentrated into 26 housing estates on the island, Fig. 2. Because of this, the infrastructure for EV becomes important.

In 2012, the Land Transport Authority of Singapore reported a total of 969910 vehicles on Singaporean roads; out of which 648221 (Approximately 67\%) are cars [22]. "Going green" in modern Singapore would including converting a significant portion of these cars to EV as long as it is economically justifiable, and Singapore has the adequate supporting infrastructure to handle this switching. Resolving these concerns involves an understanding of vehicular movements around Singapore. However, to date there is no publicized census data for this.

The distribution of the labour force on the island is given in Singapore manpower statistics [18]. Therefore, it is possible to model the movements based on these assumptions:

- Each driver stays in 1 of the 26 designated estates

- Each driver fits into manpower distribution described

- Each driver either works in the Central Business District (CBD) or elsewhere

The CBD of Singapore is located on the southern part of the island. If a driver does not work in there, then he is equally likely to be working any other parts of the island.

To model the movement, we assume that each driver stays in 1 of the 26 designated estates and has to work, which may require the driver to travel to multiple locations. Each driver will return home after work each day. Hence, each driver is associated with a set of locations

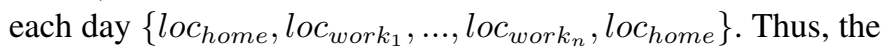
daily driving pattern can be built upon this information. To provide the notion of time, it can be further assumed that regular work hours with some flexibility to the clocking-in and out time are in placed. Work generally starts at 7 AM and ends any time after 5 PM.

As the population is not spread evenly amongst the estates, nor are they of equivalent size, the contribution of drivers from each of it would thus be different. Moreover, majority of Singaporean drivers age-ranges between 29-50 [18]. Hence, it can be assumed that the people in that age group is representative of the drivers' population for their respective estates.
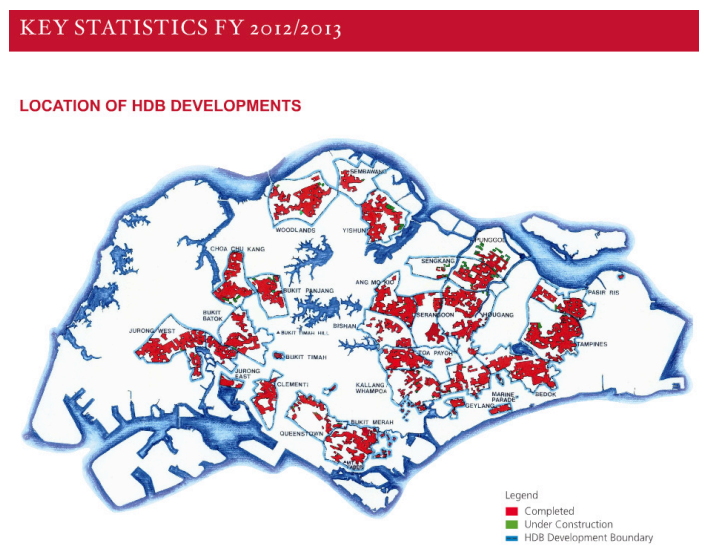

Fig. 2. Residential developments in Singapore - Sourced from key statistics of the HDB Annual Report 2012/2013 


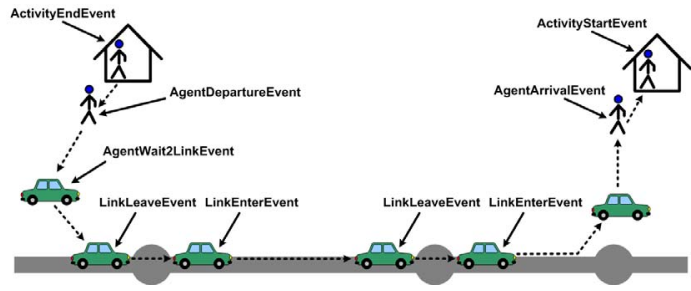

Fig. 3. Image extracted from MATSim webpage - level of granularity of events

Putting everything together, the traffic plan for one driver can be randomized by considering the following:

- Start and end its plan at its home location

- Work either in or out of the CBD area

- May travel to multiple and other locations during work

- The departure time for the next location is successive

The plans building involves various combinations of the above criteria. And this will be used as a model of the driving population of Singapore on a working day.

\section{Information Extraction}

MATSim outputs two set of data after each simulation run. The first set contains events data while the second contains link statistics data. The statistics do not serve any purpose for this research and hence it will not be touch upon any further. The events data serve as a documenter of all the actions that were taken by all the agents with high level of details including each simple and atomic action by each agent (Fig. 3). The keywords in Fig. 3 are exactly the events documented in this output. All the events are time-stamped. This facilitates the calculation of the time-spent on the roads. The event data are filtered using JAVA SAX parser to extract relevant information that contains the time used to travel the road segment of each car agent.

\section{Energy Consumption Formulation}

There are two components of energy consumption that are of interest in this study. The first is the kinetic energy consumption of the EV; energy consumed based solely on moving from point $a$ to point $b$. The second is the potential energy consumption of the EV; all the electrical components that consume energy while the EV is in used.

Suppose there exists a closed network road system that can be represented as a graph $S=(V, E)$ where $V$ are its vertices and $E$ its edges. The total amount of energy consumed, $E_{\text {Total }}$ by the EV can thought as the total amount of energy required to travel all the edges, $E_{\text {Link }_{i}}$ between the closest vertex to the start point and the closest vertex to the end point:

$$
E_{\text {Total }}=\sum_{i=1}^{n} E_{\text {Link }_{i}}
$$

where $E_{\text {Link }_{i}}$ is the energy consumed to travel the $i t h$ edge in $S$. As expressed earlier, $E_{L i n k_{i}}$ is made up of two components, the kinetic, $E_{k}$ and potential, $E_{p}$

$$
E_{\text {Link }_{i}}=E_{K_{i}}+E_{P_{i}}
$$

There are several approaches that can be used to calculate the energy consumed by EV; mostly based on calculating fuel consumption by internal combustion engine vehicles (ICEV) [23]. However, since the main topic of interest does not lie in the physical forces governing the motion of a car, a simpler yet still accurate approach is used. It is similar to [7] where a known EV's mileage parameters is used to estimate the amount of energy consumed. A more accurate and interesting option is to calculate in terms of actual fossil fuel consumption as proposed in [23], which will be reserved for the future works of this research.

The Nissan Leaf [24] is an EV that is known to have good energy efficiency. It consumes an amount 34 Megawatthours $(\mathrm{MwH})$ to travel 161 Kilometres $(\mathrm{km})$ or 0.21 Kilowatthours $(\mathrm{KwH})$ per meter. This is the average mileage given the dynamics between the physics of the Nissan Leaf and various road conditions. Hence, to calculate the kinetic component:

$$
E_{K_{i}}=\frac{0.21 \times D_{i} \times T_{i}}{3600}
$$

where $D_{i}$ is the distance of $\operatorname{Link}_{i}, T_{i}$ is the time taken to travel $\operatorname{Link}_{i}$. The unit of $E_{K_{i}}$ is in Kilojoules (KJ).

The air-conditioning (AC) unit is the main contributor of energy consumption in an EV [25]; contributing towards the potential component. It contributes toward overall expenditure with a factor of two and/or above [26]. This energy is used to offset the differences between the comfortable (internal) and the ambient (external) temperatures of an EV [27]. This is done by either heating or cooling the air intake (work Input) from the outside before being circulated into the internal hub of the EV (cooling load) as shown in Fig .4. Based on [27], the potential component can be given as:

$$
E_{P_{i}}=\left(c \rho v\left|T_{C_{i}}-T_{A_{i}}\right|\right) \cdot T_{i}
$$

where $c$ is the specific heat capacity of air, $\rho$ is the density if air, $v$ is the volume of air intake per second $T_{C_{i}}$ is the comfortable temperature that the driver maintains in the EV and $T_{A_{i}}$ is the ambient temperature at which the $\mathrm{EV}$ is travelling through $\operatorname{Link}_{i}$. Hence, the total consumption can

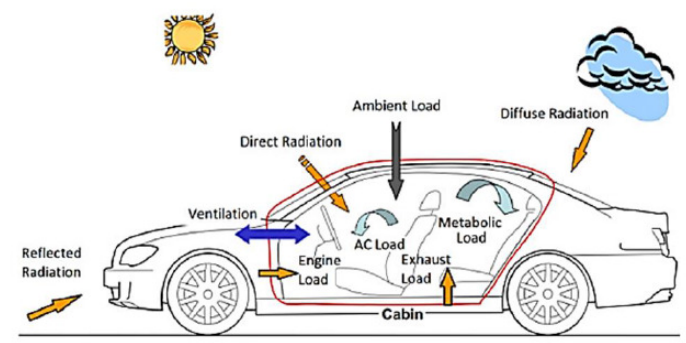

Fig. 4. Cooling of internal hub of EV against the various heating elements 


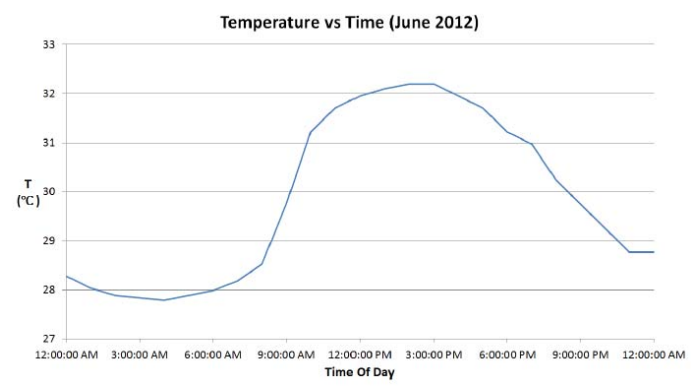

Fig. 5. Temperature inputs for AC energy consumption calculation

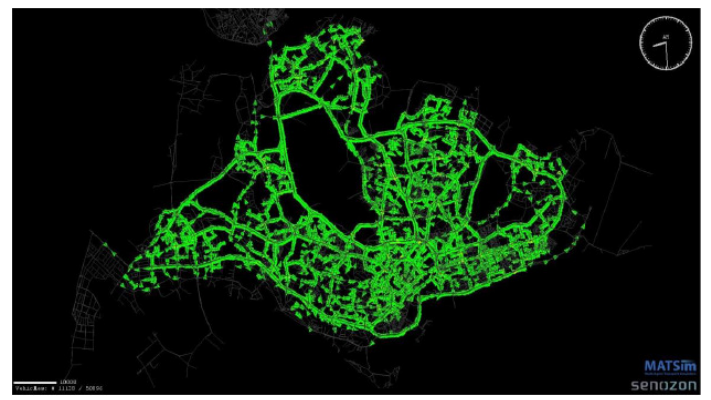

Fig. 6. Visualization of a snapshot of the simulation on MATSim's proprietary viewer; green represents the EV movements on Singapore roads

be calculated as:

$$
\begin{aligned}
E_{\text {Total }} & =\sum_{i=1}^{n} E_{\text {Link }_{i}} \\
& =\sum_{i=1}^{n}\left[E_{K_{i}}+E_{P_{i}}\right] \\
& =\sum_{i=1}^{n}\left[\frac{0.21 \times D_{i} \times T_{i}}{3600}+\left(c \rho v\left|T_{C_{i}}-T_{A_{i}}\right|\right) \cdot T_{i}\right] \\
& =\sum_{i=1}^{n} T_{i} \cdot\left[\frac{0.21 \times D_{i}}{3600}+\left(c \rho v\left|T_{C_{i}}-T_{A_{i}}\right|\right)\right]
\end{aligned}
$$

The total amount of energy required by all EV would be the summation of the energy consumed by every single trip in the simulation.

\section{Simulation}

In the simulation, $20 \%$ of the population is assumed to be working in the CBD. Due to hardware limitation, it is infeasible to simulate all the cars in Singapore using our current hardware systems. Hence, approximately $10 \%$ of the total EV population is simulated on MATSim. However, the calculated figures from the simulation will be extrapolated linearly in the final analysis. The total number of agents, representing the EV, in the simulation is 60496. The temperatures used for the AC calculation is shown in Fig 5. And the comfortable temperature of the EV is approximately $23^{\circ} \mathrm{C}$. The events data from MATSim was processed and the relevant information extracted for the energy consumption computation. All the link events pertaining to the links used by the agents in the simulation were extracted for the calculation. A dual core Intel Xeon 2.67
GHz processor with 32 Gigabytes RAM is used to power the MATSIm simulation. Running time for this simulation took 15 minutes. A snapshot of the visualization of the simulation outputs is shown in Fig. 6.

\section{A. Energy Consumption}

The results were calculated as with the formula described. The energy consumption from AC is illustrated in Fig. 7, which is in accordance with the input temperatures shown in Fig. 5. The steep increase in energy consumption is due to the majority of the EV commuting to work within a short time frame. The sudden decrease at 18th period, 9 AM, is due to many EV stopping at the CBD area for work, which is consistent with the behaviours of CBD workers.

The energy required for AC peaks at 24th period, 12 PM. This is expected as the temperature of the day is at its highest at that time. As shown in Fig. 8, this requirement is decreased by $8.44 \mathrm{MW}$ if the EV's mean comfortable temperature is increased by $3{ }^{\circ} \mathrm{C}$, which suggests that the $\mathrm{AC}$ unit does have an significant impact on the total energy consumed by EV.

\section{B. Energy Charging Pattern}

In this paper, it is assumed that the EV will be charged using a level two charging system [28] and that if it is stopped for more than two hours. [29]. The charging rate is given as per the Nissan Leaf specifications; 1.2 KW [24]. As illustrated in Fig. 9, the charging load increases as more EV arrives home

\section{Energy Consumed (Travelling)}

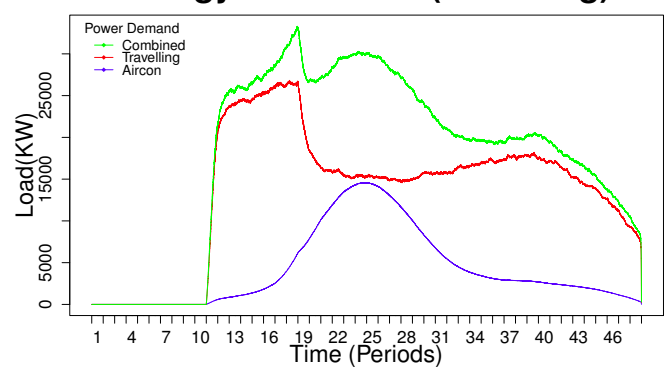

Fig. 7. Comparison of total energy consumed by EV with and without airconditioning on a per half hourly period basis starting from 0000 Hours

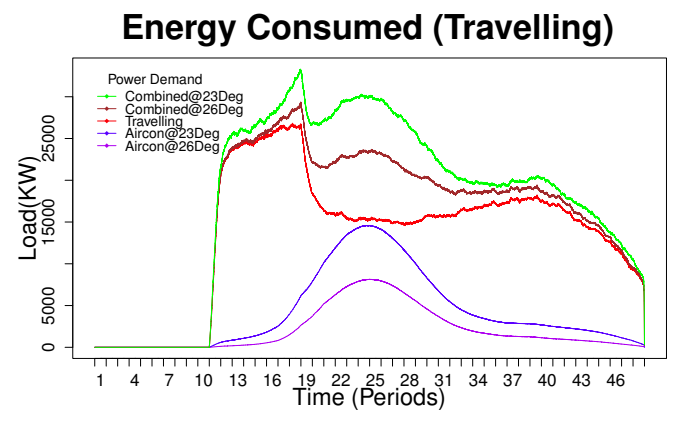

Fig. 8. Comparison of total energy consumed by EV with and without airconditioning on a per half hourly period basis starting from 0000 Hours 


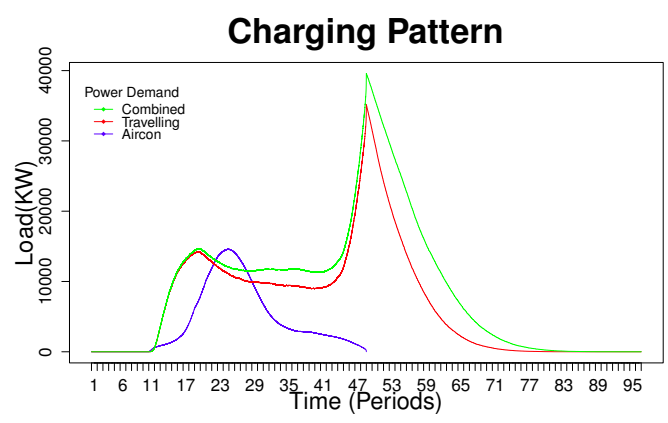

Fig. 9. Comparison of charging forecast at $1.2 \mathrm{KW}$ for $\mathrm{EV}$ with and withou air-conditioning on a per half hourly period basis starting from 0000 Hours

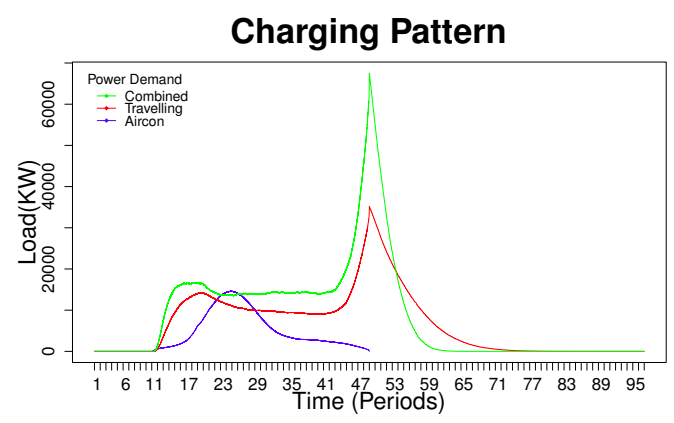

Fig. 10. Comparison of charging forecast at $3 \mathrm{KW}$ for $\mathrm{EV}$ with and withou air-conditioning on a per half hourly period basis starting from 0000 Hours

in the evening. This load peaks on 12 midnight at $39.57 \mathrm{MW}$ or $35.17 \mathrm{MW}$ if the AC load is not considered. However, it is slow for the batteries to be charged at $1.2 \mathrm{KW}$. The charging time for the last EV to be charged fully comes closed to the end of the second day. Charging at $3 \mathrm{KW}$ will ensure all EV be fully charged by the 65 th period, i.e., by 8.30 AM the next morning as shown in Fig. 10. This is more reasonable as the last EV would have been fully charged before it starts its next day's travelling routine. However, this increases the same peak load by an additional 27.96 MW. Likewise, the load can also be managed at the EV's end by encouraging the use of a higher mean comfortable temperature as shown in Fig 11 , which suggests an increase of $3{ }^{\circ} \mathrm{C}$ is able to reduce the charging time significantly.

\section{Impacts to Electricity Distribution System}

A snapshot of the half-hourly energy demand of an average day in Singapore is shown in Fig. 12. By extrapolating the data in Fig. 10 linearly to $100 \%$ of EV, an outlook into the close to actual EV charging pattern is produced. Adding this extrapolated data onto daily energy demand, the aggregated load pattern of daily usage and EV is shown in Fig. 13. Singapore has an installed generation capacity of $10477.5 \mathrm{MW}$ [2]. From Fig. 13, it is seen that at all periods it sits well below $10000 \mathrm{Mw}$. Moreover, even as the charging pattern peaks at midnight or the 48th period, the total load is below $5000 \mathrm{MW}$. Therefore, Singapore is capable of handling the impacts of the energy load generated by the EV from the generation side point of view.

\section{CONCLUSION}

In this paper, the modelling of the movement of the Singapore traffic was demonstrated. With MATSim, a MAS simulating traffic, this movement was further evolved into the driving patterns. From it, the information with regards to the time spent travelling on the roads was used to calculate the total kinetic energy consumed by the EV. Using the time spent information again but with air-conditioning model, the total potential energy consumed by the EV while travelling is calculated. It was also demonstrated by how various load pattern can be derived by simulation different parameters using MATSim.

Currently, the system is only able to produce the aggregated charging load as a whole. However, due to the mobility of EV the charging load may be aggregated unevenly at different spatial locations, which may cause problems if it is close or exceeds the capability of the system. In the future works of this research, the spatial load generated by the EV according to districts will be analysed in combination with power flow analysis to understand the impacts of the EV charging load to the infrastructure electricity grid.

\section{REFERENCES}

[1] I. ENCY, "Key world energy statistics,"

[2] Research and S. Unit, "Singapore energy statistics 2013," Energy Market Authority of Singapore, 2013.

[3] S. Shafiee and E. Topal, "When will fossil fuel reserves be diminished?," Energy Policy, vol. 37, no. 1, pp. 181-189, 2009.

[4] J. Twidell and A. D. Weir, Renewable energy resources. Taylor \& Francis, 2006.

[5] S. D. Jenkins, J. R. Rossmaier, and M. Ferdowsi, "Utilization and effect of plug-in hybrid electric vehicles in the united states power grid," in Vehicle Power and Propulsion Conference, 2008. VPPC'08. IEEE, pp. 15, IEEE, 2008.

[6] Q. Wu, A. H. Nielsen, J. Østergaard, S. T. Cha, F. Marra, Y. Chen, and C. Træholt, "Driving pattern analysis for electric vehicle (ev) grid integration study," in Innovative Smart Grid Technologies Conference Europe (ISGT Europe), 2010 IEEE PES, IEEE, 2010.

[7] S. Acha, K. H. van Dam, and N. Shah, "Modelling spatial and temporal agent travel patterns for optimal charging of electric vehicles in low carbon networks," in Power and Energy Society General Meeting, 2012 IEEE, pp. 1-8, IEEE, 2012.

[8] A. M. Uhrmacher and D. Weyns, Multi-Agent systems: Simulation and applications. CRC press, 2010.

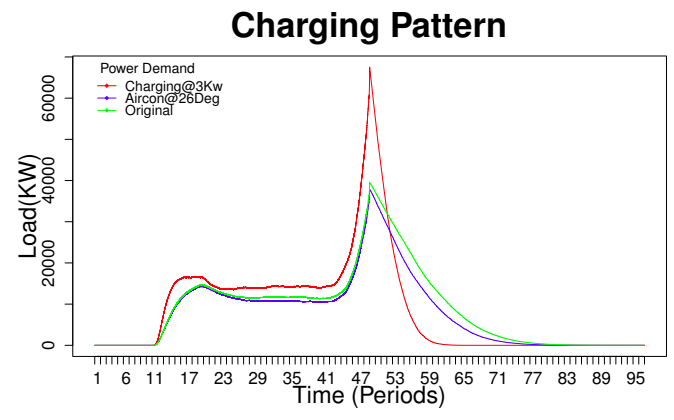

Fig. 11. Managing charging forecast by various means on a per half hourly period basis starting from 0000 Hours 


\section{Real-Time Prices and Demand - 08 Jul 14}

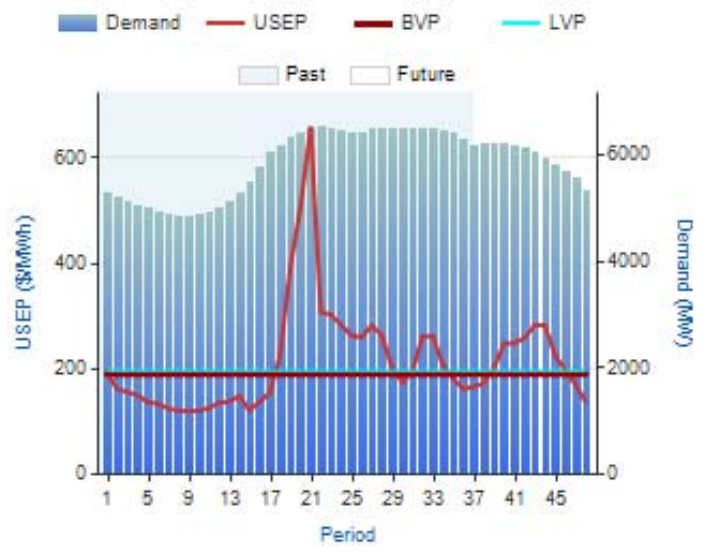

Fig. 12. Daily energy consumption and forecast from the energy market of Singapore on a per half hourly period basis starting from 0000 Hours

\section{Energy Demand}

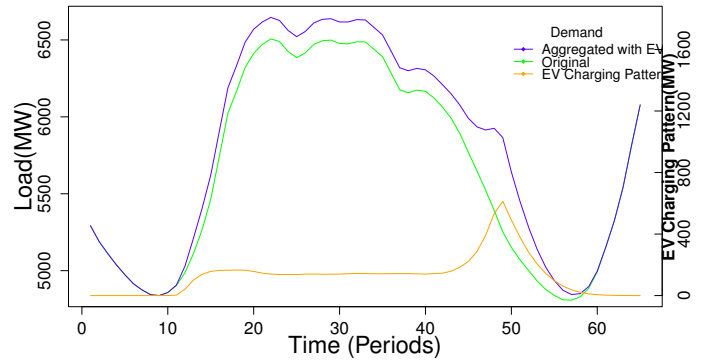

Fig. 13. Comparison of regular energy demand against a combined demand with EV on a half-hourly period

[9] R. J. Allan, Survey of agent based modelling and simulation tools Science \& Technology Facilities Council, 2010.

[10] C. F. Daganzo and Y. Sheffi, "On stochastic models of traffic assignment," Transportation Science, vol. 11, no. 3, pp. 253-274, 1977.

[11] E. W. Dijkstra, "A note on two problems in connexion with graphs," Numerische mathematik, vol. 1, no. 1, pp. 269-271, 1959.

[12] J. Ferber, Multi-agent systems: an introduction to distributed artificial intelligence, vol. 1. Addison-Wesley Reading, 1999.
[13] D. Krajzewicz, G. Hertkorn, C. Rössel, and P. Wagner, "Sumo (simulation of urban mobility)," in Proc. of the 4th middle east symposium on simulation and modelling, pp. 183-187, 2002.

[14] Q. Yang and H. N. Koutsopoulos, "A microscopic traffic simulator for evaluation of dynamic traffic management systems," Transportation Research Part C: Emerging Technologies, vol. 4, no. 3, pp. 113-129, 1996.

[15] J. Miller and E. Horowitz, "Freesim-a free real-time freeway traffic simulator," in Intelligent Transportation Systems Conference, 2007. ITSC 2007. IEEE, pp. 18-23, IEEE, 2007.

[16] R. A. Waraich, D. Charypar, M. Balmer, K. W. Axhausen, R. A Waraich, R. A. Waraich, K. W. Axhausen, and K. W. Axhausen, Performance improvements for large scale traffic simulation in matsim. ETH, Eidgenössische Technische Hochschule Zürich, IVT, Institut für Verkehrsplanung und Transportsysteme, 2009.

[17] X. From, "Schema to relations: A cost-based approach to xml storage," in Proceedings of the 18th International Conference on Data Engineering, p. 64, 2002

[18] S. Singapore, "Yearbook of statistics singapore 2013," Statistics Singapore, Singapore, 2013.

[19] Housing and D. B. (Singapore), "Public housing in singapore," September 2013.

[20] J. T. Ooi and S.-T. Lee, "Price discovery between residential land \& housing markets," Journal of Housing Research, vol. 15, no. 2, pp. 95 $112,2004$.

[21] D. of Economic and U. N. Social Affairs Population Division, "World population prospects, the 2012 revision," June 2013.

[22] S. L. Transport, "Statistics in brief," Land Transport Authority of Singapore (http://www. lta. gov. sg), 2013.

[23] E. Nam and R. Giannelli, "Fuel consumption modeling of conventional and advanced technology vehicles in the physical emission rate estimator (pere), draft," US Environmental Protection Agency, EPA420-P-05-001, 2005.

[24] Y. Sato, S. Ishikawa, T. Okubo, M. Abe, and K. Tamai, "Development of high response motor and inverter system for the nissan leaf electric vehicle," tech. rep., SAE Technical Paper, 2011.

[25] V. H. Johnson, "Fuel used for vehicle air conditioning: a state-by-state thermal comfort-based approach," tech. rep., SAE Technical Paper, 2002.

[26] B. A. Cullimore and T. J. Hendricks, "Design and transient simulation of vehicle air conditioning systems," tech. rep., SAE Technical Paper, 2001.

[27] C.-L. Li, S.-L. Chung, and J.-N. Lee, "Modeling of automobile air conditioning systems," in Control and Decision Conference, 2009. CCDC'09. Chinese, pp. 974-979, IEEE, 2009.

[28] K. Morrow, D. Karner, and J. Francfort, "Plug-in hybrid electric vehicle charging infrastructure review," US Department of Energy-Vehicle Technologies Program, 2008.

[29] C. Thomas, "Fuel cell and battery electric vehicles compared," international journal of hydrogen energy, vol. 34, no. 15, pp. 6005-6020, 2009. 